\title{
Energy efficient coordinated precoding design for a multicell system with RF energy harvesting
}

\author{
Shiwen He*, Yongming Huang, Wenyang Chen, Shi Jin, Haiming Wang and Luxi Yang
}

\begin{abstract}
This paper investigates multicell multiuser downlink systems with simultaneous wireless information and power transfer. We study the energy efficient transmission design for maximizing the system energy efficiency of data transmission (bits/Joule delivered to the receivers) which is defined as the ratio of the weighted sum rate to the total power consumption. In particular, we focus on the energy efficient coordinated precoding for the downlink of multicell system using the power splitting technique where each receiver divides the received signal into two power streams for concurrent information decoding and energy harvesting. To solve the non-convex optimization problem under consideration, first, we transform the primal optimization problem in fractional form into a tractable parameterized subtractive form optimization problem. Then, the tractable problem is solved by applying the technique of the Lagrangian optimization technique and semidefinite relaxation. An efficient solution to the considered optimization problem is finally developed. Numerical results validate the effectiveness of the proposed algorithm and reveal that the proposed algorithm can achieve better energy efficiency performance than the traditional energy efficient transmission.
\end{abstract}

Keywords: Energy harvesting; Power splitting; Energy efficient optimization; Coordinated multicell precoding; Non-convex optimization

\section{Introduction}

With explosive growth of high-data-rate applications, rapidly increasing energy is consumed in wireless networks to guarantee quality of service. Driven by this observation, energy-efficient communications have attracted increasing attention in both industry and academia [1]. In [2], the authors discussed the models of current energy consumption in base station (BS) devices. A survey of energy-efficient wireless communications can be found in [3] where several different energy efficiency metrics have been described, with 'bits per Joule' as the most popular one, which is defined as the system throughput achieved with unit-energy consumption. Later, [4] provided a general framework for obtaining the energyefficient power allocation strategy in fading channels.

*Correspondence: shiwenhe@seu.edu.cn

School of Information Science and Engineering, Southeast University, Nanjing 210096, China
Particularly, it was revealed that as the rate of the system is a concave function of the transmission power, a unique global maximum energy efficiency exists that can be achieved through an optimum power allocation strategy using fractional programming. The Pareto boundary of the achievable energy efficiency region of the $K$-links interference channels was characterized by maximizing the per-user energy efficiency which is defined as the achievable data rate at the user divided by the total power consumption of the transmitter [5]. Recently, the energy efficient coordinated beamforming optimization problem was investigated for multicell downlink systems [6-8]. In addition, considering nakagami- $m$ flat-fading channels with a delay-outage probability constraint, an energyefficient power allocation scheme was proposed in [9].

It is worth noting that all algorithm obtained in the mentioned references only focus on the energy efficiency optimization without taking the energy harvesting

\section{Springer}

(c) 2015 He et al: licensee Springer. This is an Open Access article distributed under the terms of the Creative Commons Attribution License (http://creativecommons.org/licenses/by/4.0), which permits unrestricted use, distribution, and reproduction in any medium, provided the original work is properly credited. 
into account. On the other hand, wireless power transfer technology, which enables the receivers to scavenge energy from propagating electromagnetic waves (EM) in radio frequency $(\mathrm{RF})$, has recently gained much attention in both industry and academia [10-17]. A unified study on simultaneous wireless information and power transfer (SWIPT) was presented for multiple-input multiple-output (MIMO) wireless broadcast system [10]. A SWIPT algorithm was developed for multiuser multiple input single ouptut (MISO) broadcast systems in which each of which implements information decoding (ID) or energy harvesting (EH) [11]. The authors in [12] proposed a dynamic power splitting (DPS) scheme to achieve SWIPT. Recently, the power minimization problem for simultaneous wireless information and power transfer was addressed for MISO downlink transmission systems $[13,14]$. In addition, resource allocation strategies were also investigated for a point-to-point wireless communications system with hybrid energy sources consisting of an energy harvester and a conventional energy source [15]. More recently, the resource allocation problem was solved for a point-to-point single user system with power splitting receiver in ergodic fading channels [16]. In addition, a resource allocation scheme for maximizing the energy efficiency of data transmission was developed for single input single output (SISO) frequency division multiple access (OFDMA) system with simultaneous wireless information and power transfer [17]. Whereas, it was solved based on the assumption of high signal to interference ratio and ignoring the interuser interference. It is worth mentioning that most of the work in the literature focuses on the power minimization problem with simultaneous wireless information and power transfer or the energy efficiency optimization problem for single cell SISO OFDMA communication systems. There are few work investigating the transmission design for the more complex multi-antenna multicell system.

In this paper, we investigate coordinated beamforming design for simultaneous wireless information and power transfer in multicell multiuser MIMO (MUMIMO) downlink systems. Particularly, we focus on maximizing the system energy efficiency of data transmission (bits/Joule delivered to the receivers), which is defined as the ratio of the weighted sum rate to the total power consumption, by splitting the received signal power into two parts for concurrent information decoding and $\mathrm{EH}$. The considered optimization is nonconvex and hard to tackle; to solve it, we first transform the original objective function in fractional form into a parameterized subtractive form optimization problem $[18,19]$. Then, we develop an iterative algorithm to reach its solution by alternating optimization. The convergence of the proposed algorithm is guaranteed by the monotonic boundary theorem [20]. Finally, numerical results validate the effectiveness of the proposed algorithm and show that the optimal energy efficiency and spectral efficiency can be achieved simultaneously in the low SNR region; while in the middle-high SNR region, the maximization of spectral efficiency usually cannot bring optimal energy efficiency at the same time and vice versa. Compared to the energy efficiency performance achieved by the traditional energy efficient transmission [6-8], the proposed algorithm can achieve better energy efficiency performance.

The rest of this paper is organized as follows. The system model and problem formulation are described in Section 2. In Section 3, a useful transformation of objective function is given by using jointly the relation between the minimum mean square error and the fractional programming method. Then an energy efficient precoding method with energy harvesting is developed in Section 4. The simulation results are shown in Section 5 and conclusions are finally provided in Section 6.

The following notations are used throughout this paper. Bold lowercase and uppercase letters represent column vectors and matrices, respectively. $|\boldsymbol{A}|$ denotes the determinant of matrix $A$. The superscripts $(\cdot)^{H}$, and $(\cdot)^{-1}$ represent the conjugate transpose operator, and the matrix inverse, respectively. $\operatorname{Tr}(\boldsymbol{A})$ represents the trace of matrix $\boldsymbol{A}$, and $\log (\cdot)$ is the logarithm with base $e$. The probability density function (pdf) of circular complex Gaussian random vector with mean $\boldsymbol{\mu}$ and covariance matrix $\boldsymbol{\Sigma}$ is denoted as $\mathcal{C N}(\boldsymbol{\mu}, \boldsymbol{\Sigma}) . \mathbb{E}[\cdot]$ and $\mathbb{C}$ are the statistical expectation and the complex number field, respectively.

\section{System model and problem formulation}

Consider a $K$-cell MU-MIMO downlink system where each cell includes one multiple antenna BS and a plurality of multiple antenna users. This paper focuses on a coordinated beamforming design under the assumption of perfect channel state information (CSI) knowledge at BS. We denote the $k$ th user in cell- $j$ as User- $(j, k)$ and the BS in cell- $m$ as BS- $m$. BS- $j$ is equipped with $M_{j}$ transmit antennas and serves $I_{j}$ users in cell- $j$. User- $(j, k)$ is equipped with $N_{j, k}$ antennas. The received signal of User- $(j, k)$ is then expressed as,

$$
\boldsymbol{y}_{j, k}=\sum_{m=1}^{K} \boldsymbol{H}_{m, j, k} \sum_{n=1}^{I_{m}} \boldsymbol{W}_{m, n} \boldsymbol{x}_{m, n}+\boldsymbol{z}_{j, k}
$$

where $\boldsymbol{H}_{m, j, k} \in \mathbb{C}^{N_{j, k} \times M_{m}}$ denotes the flat fading channel matrix of coefficients between BS- $m$ and User- $(j, k)$, including both the large scale fading and the small scale fading, $W_{j, k} \in \mathbb{C}^{M_{j} \times d_{j, k}}$ denotes the beamforming matrix for User- $(j, k), d_{j, k}$ denotes the number of data streams for 
User- $(j, k), \boldsymbol{x}_{j, k} \in \mathbb{C}^{d_{j, k} \times 1}$ denotes the information signal intended for User- $(j, k)$ with pdf $p\left(\boldsymbol{x}_{j, k}\right)=\mathcal{C N}(\mathbf{0}, \boldsymbol{I})$, and $\boldsymbol{z}_{j, k} \in \mathbb{C}^{N_{j, k} \times 1}$ denotes the additive white Gaussian noise (AWGN) with distribution $\mathcal{C N}\left(\mathbf{0}, \sigma_{j, k}^{2} \boldsymbol{I}\right)$. We assume that the signals for different users are independent from each other and the receiver noise. For notational convenience, let $\boldsymbol{W}_{j}=\left\{\boldsymbol{W}_{j, 1}, \cdots, \boldsymbol{W}_{j, I_{j}}\right\}$ denote the multiuser precoder set of BS- $j$ and let $W=\left\{W_{1}, \cdots, W_{K}\right\}$ denote the collection of all the precoders.

In this paper, we assume that each user applies power splitting to coordinate the process of information decoding and energy harvesting from the receive signal $[10,12]$. In particular, as shown in Figure 1, the received signal at each user is split to the information decoder and the energy harvester by a power spitter. Let $\rho_{j, k} \in(0,1)$ denote the power splitting parameter for User- $(j, k)$, this means that $100 \rho_{j, k} \%$ of the received power is used for data detection while the remaining amount is the input to the RF-EH circuity. As a result, the instantaneous rate $r_{j, k}$ of User- $(j, k)$ can be calculated as

$$
r_{j, k}=\log \left|\boldsymbol{I}+\rho_{j, k} \boldsymbol{H}_{j, j, k} \boldsymbol{W}_{j, k} \boldsymbol{W}_{j, k}^{H} \boldsymbol{H}_{j, j, k}^{H} \boldsymbol{R}_{j, k}^{-1}\right|
$$

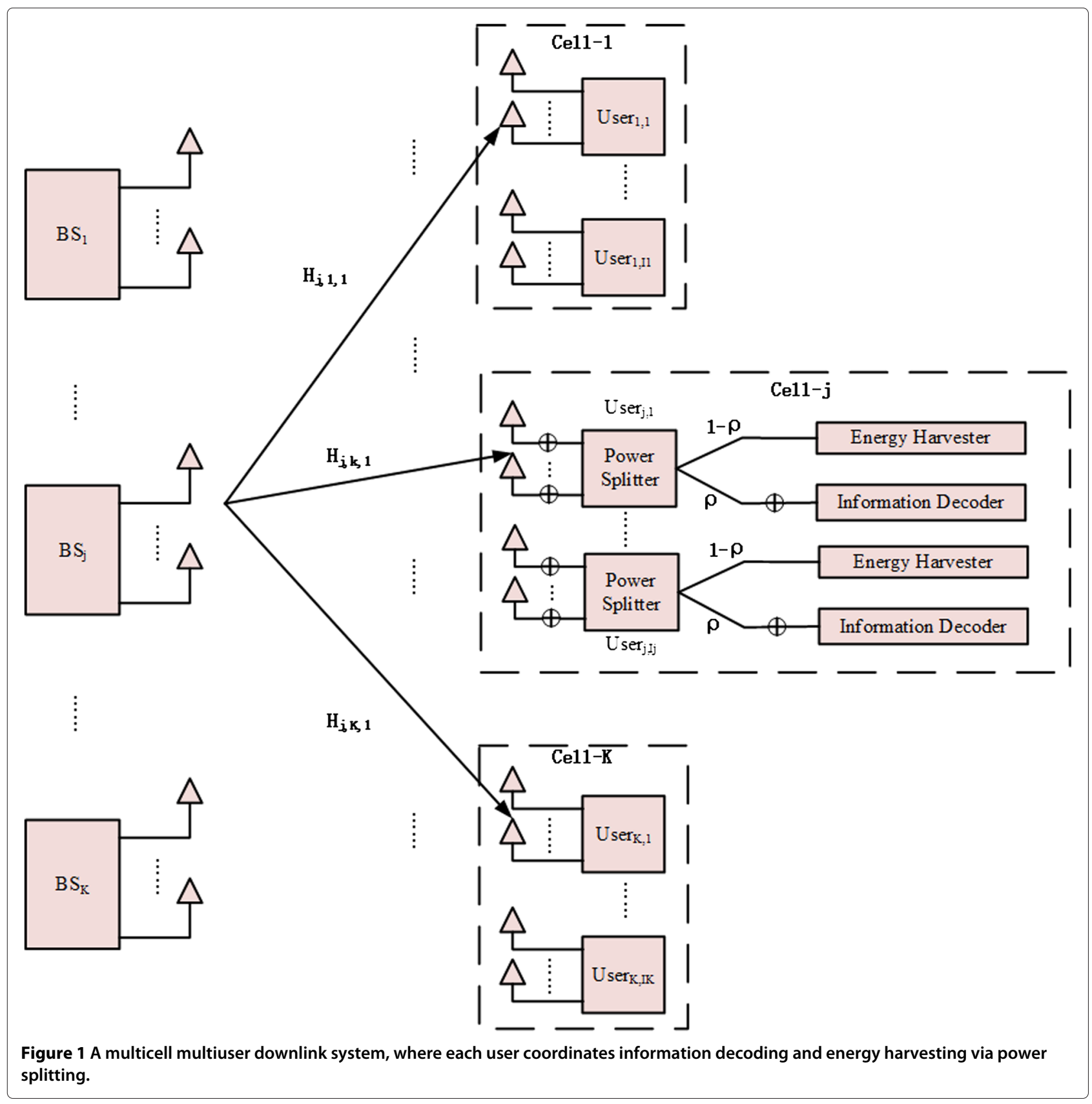


where $\boldsymbol{R}_{j, k}$ denotes the interference-plus-noise which includes the inter-user interference, the inter-cell interference and the AWGN, given by

$$
\boldsymbol{R}_{j, k}=\rho_{j, k}\left(\sum_{(m, n) \neq(j, k)} \boldsymbol{H}_{m, j, k} \boldsymbol{W}_{m, n} \boldsymbol{W}_{m, n}^{H} \boldsymbol{H}_{m, j, k}^{H}+\sigma_{j, k}^{2} \boldsymbol{I}\right)+\sigma_{s, j, k}^{2} \boldsymbol{I},
$$

where $\sigma_{s, j, k}^{2}$ is the signal processing noise power at User$(j, k)$ that is because additional circuit noise is present due to phase offsets and non-linearities which is usually modeled as AWGN with zero mean and variance $\sigma_{s, j, k}^{2}$. In order to obtain a tradeoff between the sum rate and the total power consumption, we adopt a new energy efficiency optimization criterion defined as ${ }^{\mathrm{a}}$

$$
\begin{gathered}
\max _{W} \frac{\sum_{j=1}^{K} \sum_{k=1}^{I_{j}} \varpi_{j, k} r_{j, k}}{\sum_{j=1}^{K}\left(\sum_{k=1}^{I_{j}}\left(p_{j, k}^{c}-p_{j, k}^{E}\right)+P_{C_{T}}+I_{j} P_{C_{R}}\right)} \\
\text { s.t. } \sum_{k=1}^{I_{j}} \operatorname{Tr}\left(\boldsymbol{W}_{j, k} \boldsymbol{W}_{j, k}^{H}\right) \leq P_{j}, p_{j, k}^{E} \geq \varrho_{j, k}, \forall j, k,
\end{gathered}
$$

where $P_{j}$ is power constraint of BS-j. $\varrho_{j, k}$ is a constant which specifies the minimum required power transfer to User- $(j, k)$ and $\varrho_{j, k}>0, \forall j, k$. In other words, in this paper, we consider a general case where all users have nozero harvested power target $t^{\mathrm{b}}$. The weight $\varpi_{j, k}$ is used to represent the priority of User- $(j, k)$, giving a larger value to the user with a higher rate requirement. The term $\sum_{k=1}^{I_{j}}\left(p_{j, k}^{c}-p_{j, k}^{E}\right)+P_{C_{T}}+I_{j} P_{C_{R}}$ denotes the power consumption for User- $(j, k)$. Specifically, $P_{C_{T}}$ is the constant signal processing circuit power consumption in the transmitters, caused by the transmit filter, the mixer, the frequency synthesizer, and the digital-to-analog converter, which are independent of the actual transmitted power. $P_{C_{R}}$ denotes the circuit power consumption in the receivers. $p_{j, k}^{c}$ denotes the power dissipation in the power amplifier at transmitter for User- $(j, k)$ which is defined as

$$
p_{j, k}^{c}=\vartheta_{j} \operatorname{Tr}\left(\boldsymbol{W}_{j, k} \boldsymbol{W}_{j, k}^{H}\right) .
$$

where $\vartheta_{j} \geq 1$ is a constant which accounts for the inefficiency of the power amplifier [17]. $p_{j, k}^{E}$ denotes the energy harvested at User- $(j, k)$ and is expressed as

$p_{j, k}^{E}=\left(1-\rho_{j, k}\right) \eta_{j, k} \operatorname{Tr}\left(\sum_{m=1}^{K} \sum_{n=1}^{I_{m}} \boldsymbol{H}_{m, j, k} \boldsymbol{W}_{m, n} \boldsymbol{W}_{m, n}^{H} \boldsymbol{H}_{m, j, k}^{H}+\sigma_{j, k}^{2} \boldsymbol{I}\right)$. where $0<\eta_{j, k} \leq 1$ is the energy harvesting efficiency of User- $(j, k)$. In addition, $p_{j, k}^{E}$ can be interpreted as the user's ability of harvesting energy from signal, interference and noise powers.

It is easy to see that the coupling of optimization variables leads to that problem (4) is non-convex and is therefore difficult to solve directly, which is the main drawback for the beamforming algorithm design. Moreover, the sum-of-ratio form in the objective function (4) makes the problem more intractable. In the rest of this paper, we focus on finding solution to the optimization problems (4).

\section{Transformation of the objective function}

In this section, we reformulate firstly the original problem into a tractable form by transforming the objective function into an optimization problem based on the minimum mean square errors (MMSE) receiver. In what follows, we treat interference as noise and consider linear receive beamforming strategy so that the estimated signal is given by

$$
\tilde{\boldsymbol{x}}_{j, k}=\boldsymbol{U}_{j, k} \tilde{\boldsymbol{y}}_{j, k}
$$

where $\boldsymbol{U}_{j, k} \in \mathbb{C}^{d_{j, k} \times M_{j}}$ denotes the linear equalizer for User- $(j, k), \tilde{y}_{j, k}$ is defined as

$$
\tilde{\boldsymbol{y}}_{j, k}=\sqrt{\rho_{j, k}}\left(\sum_{m=1}^{K} \boldsymbol{H}_{m, j, k} \sum_{n=1}^{I_{m}} \boldsymbol{W}_{m, n} \boldsymbol{x}_{m, n}+\boldsymbol{z}_{j, k}\right)+\overline{\boldsymbol{z}}_{j, k}
$$

where $\bar{z}_{j, k} \in \mathbb{C}^{N_{j, k} \times 1}$ denotes the AWGN with distribution $\mathcal{C N}\left(\mathbf{0}, \sigma_{s, j, k}^{2} \boldsymbol{I}\right)$. Under the independence assumption of $\boldsymbol{x}_{j, k}, \boldsymbol{z}_{j, k}$, and $\overline{\boldsymbol{z}}_{j, k}$, the MSE matrix $\boldsymbol{E}_{j, k}$ can be calculated as

$$
\begin{aligned}
\boldsymbol{E}_{j, k}= & \mathbb{E}_{\boldsymbol{x}, z, \bar{z}}\left\{\left(\tilde{\boldsymbol{x}}_{j, k}-\boldsymbol{x}_{j, k}\right)\left(\tilde{\boldsymbol{x}}_{j, k}-\boldsymbol{x}_{j, k}\right)^{H}\right\} \\
= & \rho_{j, k} \boldsymbol{U}_{j, k} \sum_{m=1}^{K} \sum_{n=1}^{I_{m}} \boldsymbol{H}_{m, j, k} \boldsymbol{W}_{m, n} \boldsymbol{W}_{m, n}^{H} \boldsymbol{H}_{m, j, k}^{H} \boldsymbol{U}_{j, k}^{H} \\
& +\rho_{j, k} \sigma_{j, k}^{2} \boldsymbol{U}_{j, k} \boldsymbol{U}_{j, k}^{H}+\sigma_{s, j, k}^{2} \boldsymbol{U}_{j, k} \boldsymbol{U}_{j, k}^{H} \\
& -\sqrt{\rho_{j, k}} \boldsymbol{U}_{j, k} \boldsymbol{H}_{j, j, k} \boldsymbol{W}_{j, k}-\sqrt{\rho_{j, k}} \boldsymbol{W}_{j, k}^{H} \boldsymbol{H}_{j, j, k}^{H} \boldsymbol{U}_{j, k}^{H}+\boldsymbol{I} \\
= & \rho_{j, k} \boldsymbol{U}_{j, k} \sum_{(m, n) \neq(j, k)} \boldsymbol{H}_{m, j, k} \boldsymbol{W}_{m, n} \boldsymbol{W}_{m, n}^{H} \boldsymbol{H}_{m, j, k}^{H} \boldsymbol{U}_{j, k}^{H} \\
& +\rho_{j, k} \sigma_{j, k}^{2} \boldsymbol{U}_{j, k} \boldsymbol{U}_{j, k}^{H}+\left(\boldsymbol{I}-\sqrt{\rho_{j, k}} \boldsymbol{U}_{j, k} \boldsymbol{H}_{j, j, k} \boldsymbol{W}_{j, k}\right) \\
& \times\left(\boldsymbol{I}-\sqrt{\rho_{j, k}} \boldsymbol{U}_{j, k} \boldsymbol{H}_{j, j, k} \boldsymbol{W}_{j, k}\right)^{H}+\sigma_{s, j, k}^{2} \boldsymbol{U}_{j, k} \boldsymbol{U}_{j, k}^{H}
\end{aligned}
$$

Compared with the traditional MMSE expression $[21,22]$, the new MMSE expression given in (9) includes 
more factors, such as the power splitter factor $\rho_{j, k}$ and the signal processing noise item. Fixing all the transmit beamformers $W$ and minimizing MSE lead to the well-known MMSE receiver:

$$
\boldsymbol{U}_{j, k}^{\mathrm{mmse}}=\sqrt{\rho_{j, k}} \boldsymbol{W}_{j, k}^{H} \boldsymbol{H}_{j, j, k}^{H} \boldsymbol{T}_{j, k}^{-1}
$$

where $\boldsymbol{T}_{j, k}$ is the covariance matrix of the total received signal at User- $(j, k)$ and is calculated by

$\boldsymbol{T}_{j, k}=\rho_{j, k} \sum_{m=1}^{K} \sum_{m=1}^{I_{m}} \boldsymbol{H}_{m, j, k} \boldsymbol{W}_{m, n} \boldsymbol{W}_{m, n}^{H} \boldsymbol{H}_{m, j, k}^{H}+\rho_{j, k} \sigma_{j, k}^{2} \boldsymbol{I}+\sigma_{s, j, k}^{2} \boldsymbol{I}$

Using this MMSE receiver, the corresponding MSE matrix is given by

$$
\boldsymbol{E}_{j, k}^{\text {mmse }}=\boldsymbol{I}-\rho_{j, k} \boldsymbol{W}_{j, k}^{H} \boldsymbol{H}_{j, j, k}^{H} \boldsymbol{T}_{j, k}^{-1} \boldsymbol{H}_{j, j, k} \boldsymbol{W}_{j, k}
$$

According to the Woodbury matrix identity ([23], pp. 124, 3.8.3), we have

$$
\left|\left(\boldsymbol{E}_{j, k}^{\mathrm{mmse}}\right)^{-1}\right|=\left|\boldsymbol{I}+\rho_{j, k} \boldsymbol{H}_{j, j, k} \boldsymbol{W}_{j, k} \boldsymbol{W}_{j, k}^{H} \boldsymbol{H}_{j, j, k}^{H} \boldsymbol{R}_{j, k}^{-1}\right|
$$

Combining (2) with (13) and applying the conclusion given in $[21,22]$, problem (4) can be reformulated as follows

$$
\begin{aligned}
\max _{\boldsymbol{W}, \boldsymbol{U}, \boldsymbol{\Sigma}} \frac{\sum_{j=1}^{K} \sum_{k=1}^{I_{j}} \varpi_{j, k}\left(-\operatorname{Tr}\left(\boldsymbol{\Sigma}_{j, k} \boldsymbol{E}_{j, k}\right)+\log \left|\boldsymbol{\Sigma}_{j, k}\right|+d_{j, k}\right)}{\sum_{j=1}^{K}\left(\vartheta_{j} \sum_{k=1}^{I_{j}} p_{j, k}^{c}+P_{C_{T}}+I_{j} P_{C_{R}}\right)} \\
\text { s.t. } \sum_{k=1}^{I_{j}} \operatorname{Tr}\left(\boldsymbol{W}_{j, k} \boldsymbol{W}_{j, k}^{H}\right) \leq P_{j}, p_{j, k}^{E} \geq \varrho_{j, k}, \forall j, k,
\end{aligned}
$$

where $\boldsymbol{U}=\left\{\boldsymbol{U}_{1}, \cdots, \boldsymbol{U}_{K}\right\}$ denotes the collection of all the linear equalizers and $\boldsymbol{U}_{j}=\left\{\boldsymbol{U}_{j, 1}, \cdots, \boldsymbol{U}_{j, I_{j}}\right\}$ denotes the collection of all the linear equalizers in cell-j. $\boldsymbol{\Sigma}=$ $\left\{\boldsymbol{\Sigma}_{1}, \cdots, \boldsymbol{\Sigma}_{K}\right\}$ denotes the collection of all the auxiliary variables and $\boldsymbol{\Sigma}_{j}=\left\{\boldsymbol{\Sigma}_{j, 1}, \cdots, \boldsymbol{\Sigma}_{j, I_{j}}\right\}$ denotes the collection of all the auxiliary variables in cell- $j$. Note that the equivalence relation implies that the weighted sum-rate can be replaced with a optimization form with some additional auxiliary variables, see (14). The latter problem is in the space of $(\boldsymbol{W}, \boldsymbol{U}, \boldsymbol{\Sigma})$ and is easier to handle since optimizing each variable while holding others fixed is convex and easy (e.g., closed form). In the following section, this property will be exploited to design an effective energy efficient transmission method with taking energy harvesting into account.

\section{Energy-efficient optimization algorithm with energy harvesting}

\subsection{Algorithm design}

It is easy to see that the function given by the numerator in the objective function (14) is concave in each of the optimization variables $\boldsymbol{W}, \boldsymbol{U}$, and $\boldsymbol{\Sigma}$, but the function given by the numerator in the objective function (14) is jointly non-concave with respect to variables $\boldsymbol{W}, \boldsymbol{U}$, and $\boldsymbol{\Sigma}$. In the sequel, we propose to use the block coordinate optimization method to solve (14). In particular, we optimize the problem by sequentially fixing two of the three variables $\boldsymbol{W}, \boldsymbol{U}, \boldsymbol{\Sigma}$ and updating the third. The results in ([17], Theorem 1) has revealed that there exists an equivalent optimization problem with an objective function in subtractive form for an optimization problem with an objective function in fractional form. It also means that we need to search a value of $q$ such that the optimal value of the objective function of the following problem (15) is zero.

$$
\begin{aligned}
\max _{\boldsymbol{W}, \boldsymbol{U}, \boldsymbol{\Sigma}} & \sum_{j=1}^{K} \sum_{k=1}^{I_{j}} \varpi_{j, k}\left(-\operatorname{Tr}\left(\boldsymbol{\Sigma}_{j, k} \boldsymbol{E}_{j, k}\right)+\log \left|\boldsymbol{\Sigma}_{j, k}\right|+d_{j, k}\right) \\
& -q \sum_{j=1}^{K}\left(\vartheta_{j} \sum_{k=1}^{I_{j}} p_{j, k}^{c}+P_{C_{T}}+I_{j} P_{C_{R}}\right) \\
\text { s.t. } & \sum_{k=1}^{I_{j}} \operatorname{Tr}\left(\boldsymbol{W}_{j, k} \boldsymbol{W}_{j, k}^{H}\right) \leq P_{j}, p_{j, k}^{E} \geq \varrho_{j, k}, \forall j, k,
\end{aligned}
$$

It is easily known that for fixed $q$, and $W$, the optimal solution of $\boldsymbol{U}_{j, k}^{o p t}$ is given by (10) and the optimal solution of $\boldsymbol{\Sigma}_{j, k}$ is calculated as

$$
\Sigma_{j, k}^{o p t}=\boldsymbol{E}_{j, k}^{-1}
$$

In what follows, we focus on solving problem (15) with fixed $q, \boldsymbol{U}$ and $\boldsymbol{\Sigma}$ which can be reformulated as

$$
\begin{aligned}
\min _{W} & \sum_{j=1}^{K} \sum_{k=1}^{I_{j}}\left(\varpi_{j, k} \operatorname{Tr}\left(\boldsymbol{\Sigma}_{j, k} \boldsymbol{E}_{j, k}\right)+q \vartheta_{j} p_{j, k}^{c}\right) \\
\text { s.t. } & \sum_{k=1}^{I_{j}} \operatorname{Tr}\left(\boldsymbol{W}_{j, k} \boldsymbol{W}_{j, k}^{H}\right) \leq P_{j}, p_{j, k}^{E} \geq \varrho_{j, k}, \forall j, k,
\end{aligned}
$$

Then, the Lagrangian of problem (17) is given by

$$
\begin{aligned}
\mathcal{L}(\boldsymbol{W}, \boldsymbol{\kappa}, \boldsymbol{\lambda})= & \sum_{j=1}^{K} \sum_{k=1}^{I_{j}}\left(\varpi_{j, k} \operatorname{Tr}\left(\boldsymbol{\Sigma}_{j, k} \boldsymbol{E}_{j, k}\right)+q p_{j, k}^{c}\right) \\
& +\sum_{j=1}^{K} \kappa_{j}\left(\sum_{k=1}^{I_{j}} \operatorname{Tr}\left(\boldsymbol{W}_{j, k} \boldsymbol{W}_{j, k}^{H}\right)-P_{j}\right) \\
& +\sum_{j=1}^{K} \sum_{k}^{I_{j}} \lambda_{j, k}\left(\varrho_{j, k}-p_{j, k}^{E}\right)
\end{aligned}
$$


where $\boldsymbol{\kappa}$ and $\lambda$ denote the Lagrange multipliers associated with the power constraints and the individual minimum required power transfer constraints. Thus, the dual problem for the primal problem (17) is given by

$$
\max _{\kappa, \lambda} \min _{W} \mathcal{L}(W, \kappa, \lambda)
$$

By plugging (5) and (9) into (18) and using the standard convex optimization techniques and the KKT conditions, the optimal solution of $W$ to problem (17) is given by

$$
\boldsymbol{W}_{j, k}=\varpi_{j, k} \sqrt{\rho_{j, k}} \boldsymbol{\Phi}_{j}^{-1} \boldsymbol{H}_{j, j, k}^{H} \boldsymbol{U}_{j, k}^{H} \boldsymbol{\Sigma}_{j, k}
$$

where $\boldsymbol{\Phi}_{j}$ is calculated as

$$
\begin{aligned}
\boldsymbol{\Phi}_{j}= & \sum_{m=1}^{K} \sum_{n=1}^{I_{m}} \boldsymbol{H}_{j, m, n}^{H}\left(\varpi_{m, n} \rho_{m, n} \boldsymbol{U}_{m, n}^{H} \boldsymbol{\Sigma}_{m, n} \boldsymbol{U}_{m, n}\right. \\
& \left.-\eta_{m, n}\left(q+\lambda_{m, n}\right)\left(1-\rho_{m, n}\right) \boldsymbol{I}\right) \boldsymbol{H}_{j, m, n}+\left(q+\kappa_{j}\right) \vartheta_{j} \boldsymbol{I}
\end{aligned}
$$

In addition, the outer optimization of problem (19) can be achieved by using the gradient method which leads to the following Lagrange multiplier update equations:

$$
\begin{gathered}
\kappa_{j}^{(\mu+1)}=\left[\kappa_{j}^{(\mu)}+\xi_{1}(\mu) \times\left(\sum_{k=1}^{I_{j}} \operatorname{Tr}\left(W_{j, k} W_{j, k}^{H}\right)-P_{j}\right)\right]^{+} \\
\lambda_{j, k}^{(\mu+1)}=\left[\lambda_{j, k}^{(\mu)}+\xi_{2}(\mu) \times\left(\varrho_{j, k}-p_{j, k}^{E}\right)\right]^{+}
\end{gathered}
$$

where $[x]^{+}$is defined as $[x]^{+}=\max \{0, x\}$, index $\mu \geq 0$ is the iteration index and $\xi_{i}(\mu), i=1,2$, are positive step size. Here, it should be pointed out that the initialization of the precoders $W$ can be achieved by solving the following optimization problem.

$$
\begin{aligned}
& \min _{\boldsymbol{W}} \sum_{j=1}^{K} \sum_{k=1}^{I_{j}} \operatorname{Tr}\left(\overline{\boldsymbol{W}}_{j, k}\right), \text { s.t. } \overline{\boldsymbol{W}}_{j, k} \geq 0, \operatorname{Rank}\left(\overline{\boldsymbol{W}}_{j, k}\right)=d_{j, k}, \\
& \operatorname{Tr}\left(\sum_{m=1}^{K} \sum_{n=1}^{I_{m}} \boldsymbol{H}_{m, j, k} \overline{\boldsymbol{W}}_{m, n} \boldsymbol{H}_{m, j, k}^{H}\right)+N_{j, k} \sigma_{j, k}^{2} \geq \frac{\varrho_{j, k}}{\left(1-\rho_{j, k}\right) \eta_{j, k}}, \forall j, k .
\end{aligned}
$$

where $\bar{W}_{j, k}=W_{j, k} \boldsymbol{W}_{j, k}^{H}$. It is easy to see that problem (24) can be further expressed in standard semidefined programming (SDP) form with linear matrix inequalities (see [24] Equation (1)) as formulation (25).

$$
\begin{aligned}
& \min _{\boldsymbol{W}} \sum_{j=1}^{K} \sum_{k=1}^{I_{j}} \operatorname{Tr}\left(\overline{\boldsymbol{W}}_{j, k}\right), \text { s.t. } \overline{\boldsymbol{W}}_{j, k} \geq 0, \operatorname{Rank}\left(\overline{\boldsymbol{W}}_{j, k}\right)=d_{j, k}, \\
& {\left[\begin{array}{cc}
\operatorname{Tr}\left(\sum_{m=1}^{K} \sum_{n=1}^{I_{m}} \boldsymbol{H}_{m, j, k} \overline{\boldsymbol{W}}_{m, n} \boldsymbol{H}_{m, j, k}^{H}\right)+N_{j, k} \sigma_{j, k}^{2} & \sqrt{\varrho_{j, k}} \\
\sqrt{\varrho_{j, k}} & \left(1-\rho_{j, k}\right) \eta_{j, k}
\end{array}\right] \geq 0, \forall j, k .}
\end{aligned}
$$

To make problem (25) tractable, we first ignore the rank constraints and focus on problem (25) without rank constraints named as relaxed problem. The relaxed problem is convex and belongs to the class of SDP, as it is composed of cones of positive semdefinite matrices and linear matrix inequalities involving $W$. The numerical solution can be found by using SDP solvers. Suppose $\overline{\boldsymbol{W}}^{*}$ is the optimal solution to the relaxed problem, due to the relaxation, $\operatorname{Rank}\left(\bar{W}_{j, k}^{*}\right)$ may not satisfy the corresponding rank constraint in general. To address the issues, the randomization technique is adopted here to solve the rank constraint problem. In the randomization technique, we eigendecompose $\overline{\boldsymbol{W}}_{j, k}^{*}=\overline{\boldsymbol{U}}_{j, k} \boldsymbol{\Lambda}_{j, k} \overline{\boldsymbol{U}}_{j, k}^{H}$, and choose $\overline{\boldsymbol{W}}_{j, k}=\overline{\boldsymbol{U}}_{j, k} \boldsymbol{\Lambda}_{j, k}^{1 / 2} \boldsymbol{\Psi}_{j, k} \boldsymbol{\Psi}_{j, k}^{H} \boldsymbol{\Lambda}_{j, k}^{1 / 2} \overline{\boldsymbol{U}}_{j, k}^{H}$ as the solution to problem (25), where $\boldsymbol{\Psi}_{j, k}$ is an $M_{j} \times d_{j, k}$ randomly generated matrix whose entries satisfy uniform or Gaussian distribution [25]. Then the initialization of $\boldsymbol{W}_{j, k}$ can be obtained as $\boldsymbol{W}_{j, k}=\overline{\boldsymbol{U}}_{j, k} \boldsymbol{\Lambda}_{j, k}^{1 / 2} \boldsymbol{\Psi}_{j, k}$. To summarize, one algorithm for solving problem (14) is described as Algorithm 1.

$\overline{\text { Algorithm } 1 \text { Energy efficient precoding transmission }}$ with energy harvesting

1: Set $q=0$;

2: Initialize $\boldsymbol{W}^{(v)}$ with $v=0$, let $h$ denote the objective value of (15) and let $h^{(v)}=0$;

3: Update $\boldsymbol{U}$ and $\boldsymbol{\Sigma}$ with (10), (14), and $\boldsymbol{W}^{(v)}$, and obtain $\boldsymbol{U}^{(v)}$ and $\boldsymbol{\Sigma}^{(v)}$;

4: Let $v=v+1$, update $W$ by solving problem (17) with fixed $\boldsymbol{U}^{(\nu-1)}$ and $\boldsymbol{\Sigma}^{(\nu-1)}$, and obtain $\boldsymbol{W}^{(\mu)}$;

5: Compute the objective value of (15) with $\boldsymbol{W}^{(\mu)}, \boldsymbol{U}^{(\nu-1)}$, and $\boldsymbol{\Sigma}^{(\nu-1)}$, and obtain $h^{(\nu)}$. If $\left|h^{(v)}-h^{(v-1)}\right| \leq \delta$, then go to 6 , otherwise go to step 3;

6: Update the value of $q$ as

$$
q=\frac{\sum_{j=1}^{K} \sum_{k=1}^{I_{j}} \varpi_{j, k}\left(-\operatorname{Tr}\left(\boldsymbol{\Sigma}_{j, k} \boldsymbol{E}_{j, k}\right)+\log \left|\boldsymbol{\Sigma}_{j, k}\right|+d_{j, k}\right)}{\sum_{j=1}^{K}\left(\vartheta_{j} \sum_{k=1}^{I_{j}} p_{j, k}^{c}+P_{C_{T}}+I_{j} P_{C_{R}}\right)}
$$

with $\boldsymbol{W}^{(v)}, \boldsymbol{U}^{(v-1)}$, and $\boldsymbol{\Sigma}^{(v-1)}$. If $\boldsymbol{W}^{(v)}, \boldsymbol{U}^{(v-1)}$, and $\boldsymbol{\Sigma}^{(\nu-1)}$ meets the following inequality

$$
\begin{array}{r}
\mid \sum_{j=1}^{K} \sum_{k=1}^{I_{j}} \varpi_{j, k}\left(-\operatorname{Tr}\left(\boldsymbol{\Sigma}_{j, k} \boldsymbol{E}_{j, k}\right)+\log \left|\boldsymbol{\Sigma}_{j, k}\right|+d_{j, k}\right) \\
-q \sum_{j=1}^{K}\left(\vartheta_{j} \sum_{k=1}^{I_{j}} p_{j, k}^{c}+P_{C_{T}}+I_{j} P_{C_{R}}\right) \mid \leq \delta
\end{array}
$$

then stop the iteration, otherwise go to step 2 .

Note that Algorithm 1 only aims to solve problem (4) with fixed power splitter factors $\rho$, we assume that all 
users have the same power splitter factors, namely, $\rho_{j, k}=$ $\rho, \forall j, k$. Let $E E(\rho)$ denote the objective value of problem (4) with given $\rho$. It is easy to see that $E E$ (1) denote the maximum energy efficiency value of problem (4) that only aims to maximize the system energy efficiency without taking the energy harvesting into account. Based on this results, we further develop Algorithm 2 as below to numerically search the optimal value of $\rho$.

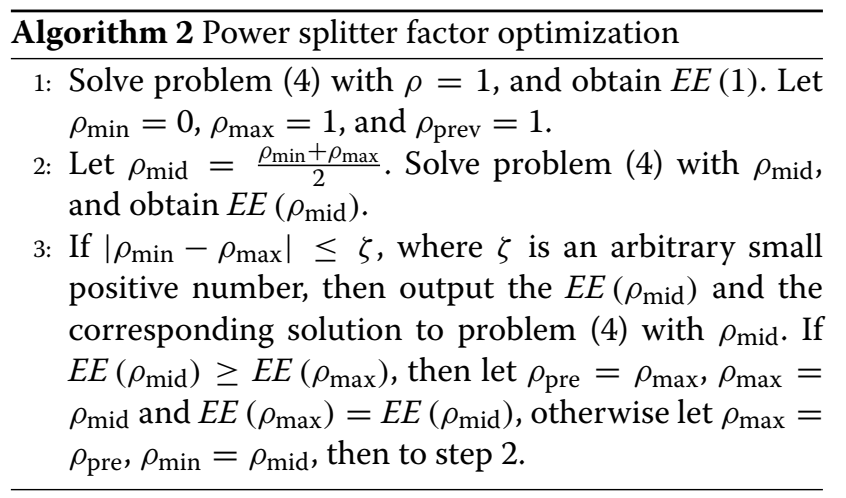

\subsection{Algorithm analysis}

In this subsection, we briefly analyze the convergence and the computational complexity of Algorithm 1.

Lemma 1. The sequence generated by the step 3 to step 4 of Algorithm 1 guarantees to converge to a stationary point of problem (15). Furthermore, the convergence of Algorithm 1 also can be guaranteed.

Proof. It is easily seen that the updates of step 3 to step 4 in Algorithm 1 all aim to increase the sum rate so that an increasing sequence is generated while the iteration running. Since the achievable SINR region under the transmit power constraint is bounded, the sum rate is also bounded. The convergence of Algorithm 1 is guaranteed by the monotonic convergence theorem [26,27]. Note that the optimization problem (15) has a differentiable objective function and the transmit power constraints only work for variable $W$. It follows from the general optimization theory [26,27] that steps 3 to 4 in Algorithm 1, which is a block coordinate ascent method applied to problem (15), converge to a stationary point of problem (15). Based on these analyses, the convergence of Algorithm 1 can be guaranteed with the fractional theorem obtained in $[18,19]$.

It is easily found that the computational complexity of Algorithm 1 mainly arise from the matrix inversion operation which is used to calculate the optimal linear equalizer given by (10), the optimal auxiliary variable obtained by (16) and the optimal beamformer based on (20), respectively. Assume that $M_{j}=M, N_{j, k}=N, I_{j}=I, d_{j, k}=d$, $\forall j, k, d \leq N$ and $I d \leq \min (I N, M)$. We count the floating point operations (flops) to indicate the complexity. A flop corresponds to a real floating-point operation. Therefore, a real addition, multiplication, or division is counted as one flop while a complex addition and multiplication have two flops and six flops, respectively. Similar to [28,29], the complexity of some basic matrix calculations are approximately counted as follows: Multiplication of two $m \times p$ and $p \times q$ complex matrices involves $8 m p q$ flops; inversion of an $m \times m$ Hermite matrix involves $\frac{4 m^{3}}{3}$ flops; Inversion of an $m \times m$ real matrix involves $\frac{2 m^{3}}{3}$ flops; inversion of an $m \times m$ real symmetric matrix involves $\frac{m^{3}}{3}$ flops. Based on these references, the computation required for each active User- $(j, k)$ is as follows:

- Inversion of $\boldsymbol{\Phi}_{j}$ needs about $\frac{4 M^{3}}{3}+8 d M K I(M+N)$ flops. Therefore, the update of the beamforming matrix $\boldsymbol{W}_{j, k}$ needs about $\frac{4 M^{3}}{3}+8 d M(K I M+K I N+d)$ flops. We also note that matrix $\boldsymbol{\Phi}_{j}$ can be calculated only once for all beamforming $\boldsymbol{W}_{j, k}, \forall k$.

- The calculation of $\boldsymbol{T}_{j, k}^{-1}$ needs about $\frac{4 N^{3}}{3}+8 d N K I(M+N)$ flops. Therefore, the calculation of $\boldsymbol{U}_{j, k}$ needs about $\frac{4 N^{3}}{3}+8 d N K I(M+N)+8 d N^{2}$ flops.

- The calculation of $\boldsymbol{\Sigma}_{j, k}$ needs about $\frac{4 d^{3}}{3}+8 d^{2} N$ flops.

Therefore, one execution of steps 3 to 4 in Algorithm 1 takes approximately $\frac{4}{3} K I\left(M^{3}+N^{3}+d^{3}\right)+$ $8 d K^{2} I^{2}(M+N)(M+2 N)+8 d M K I(K I N+d)=$ $O\left(37 K^{2} I^{2} \tau^{3}\right)$ flops, where $\tau=\max (M, N)$.

\section{Numerical results}

In this section, we numerically evaluate the performance of the proposed energy efficient transmission algorithm in multicell multiuser downlink systems. We consider a small cell communication systems with $K=3$ coordinated BSs, and assume that all users have the same set of parameters, i.e., $d_{j, k}=d, \eta_{j, k}=\eta, \rho_{j, k}=\rho, \varrho_{j, k}=\varrho$, $\sigma_{j, k}^{2}=\sigma^{2}, P_{j}=P, \sigma_{s, j, k}^{2}=\sigma_{s}^{2}, N_{j, k}=N, M_{j}=M$, and $I_{j}=I, \forall j, k$. Moreover, we set $P_{C_{T}}=30 \mathrm{dBm}, P_{C_{R}}=20$ $\mathrm{dBm}, \eta=0.5, \sigma^{2}=-70 \mathrm{dBm}, \sigma_{s}^{2}=-50 \mathrm{dBm}$ in all simulations $[14,17]$. It is further assumed that the signal attenuation from $\mathrm{BS}$ to its served user is $8 \hat{d} \mathrm{~dB}$ corresponding to an identical distance of $\hat{d}$ meters, where $6 \leq \hat{d} \leq 10$ in unit of meters, i.e, its served user locates in the cooperation and energy harvesting region, as shown in Figure 2. With this transmission distance, the line-of-sight (LOS) signal is dominant, and thus the Rician fading is used 


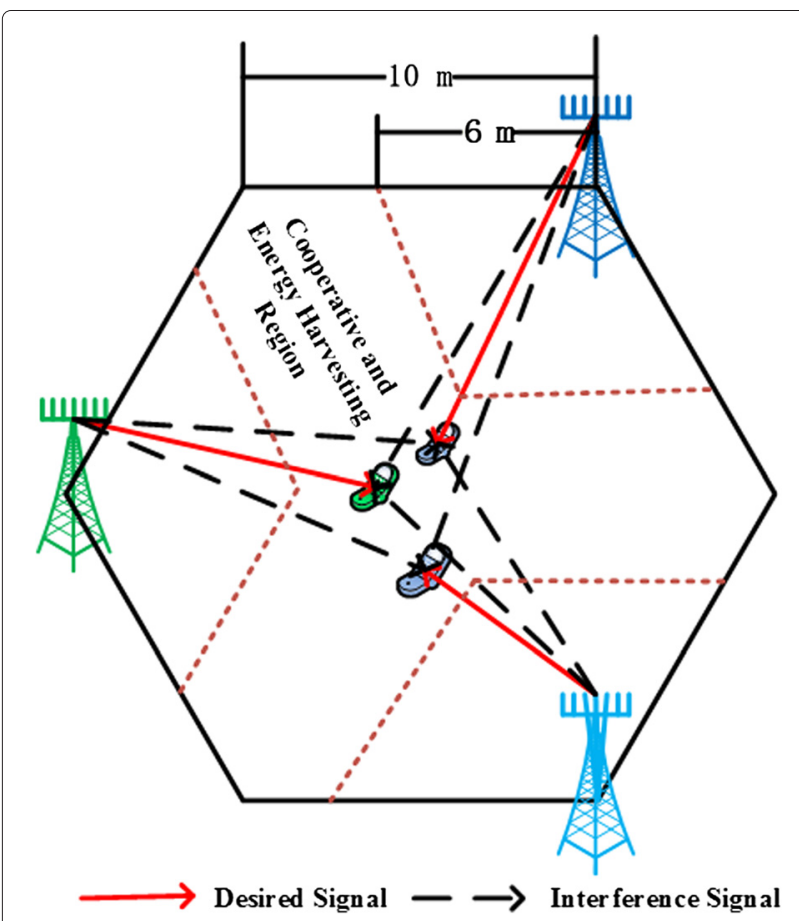

Figure 2 Simulation model.

to model the channel [30]. Specially, $\boldsymbol{H}_{m, j, k}$ is expressed as

$$
\boldsymbol{H}_{m, j, k}=\sqrt{\frac{K_{R}}{1+K_{R}}} \boldsymbol{H}_{m, j, k}^{\mathrm{LOS}}+\sqrt{\frac{1}{1+K_{R}}} \boldsymbol{H}_{m, j, k}^{\mathrm{NLOS}}
$$

where $\boldsymbol{H}_{m, j, k}^{\mathrm{NLOS}}$ denotes the Rayleigh fading component with each element being a circularly symmetric complex Gaussian random variable with zero mean and covariance $-8 \hat{d}_{m, j, k} \mathrm{~dB}, K_{R}$ is the Rician factor set to be $6 \mathrm{~dB}$, and $\boldsymbol{H}_{m, j, k}^{L O S} \in \mathbb{C}^{N \times M}$ is the LOS deterministic component and is given as ${ }^{\mathrm{c}}$

$$
\boldsymbol{H}_{m, j, k}^{\mathrm{LOS}}=10^{-\sqrt{d_{m, j, k}}}\left[\begin{array}{ccc}
e^{j \phi_{1,1}} & \cdots & e^{j \phi_{1, M}} \\
\vdots & \ddots & \vdots \\
e^{j \phi_{N, 1}} & \cdots & e^{j \phi_{N, M}}
\end{array}\right]
$$

where $e^{j \phi_{n, m}}$ are the elements of the fixed LOS matrix $\boldsymbol{H}_{m, j, k}^{\mathrm{LOS}}$ with $\phi_{n, m}=-\frac{2 \pi \iota \sin \left(\epsilon_{n, m}\right)}{\lambda}$, where $\iota$ is the spacing between successive antenna elements at BS, $\lambda$ is the carrier wavelength, and $\epsilon_{n, m}$ is derived from the angle of arrival/departure of the LOS component. For the sake of simplify, following [31], $\phi_{n, m}$ is set to be zero in all simulations, $\forall n, m$. In addition, a $12 \mathrm{~dB}$ effective antenna gain was also considered in our simulation.

For the sake of illustration, the performance curves of Algorithm 1 with fixed power splitting factor $\rho=0.8$ and Algorithm 1 with optimized power splitting factor $\rho$ are labeled as 'proposed algorithm I' and 'proposed algorithm
II' in our simulation figures, respectively. For baseline I, we maximize the weighted sum rate (bit/s/Hz) with respect to $W$ subject to per-BS power constraint, instead of the energy efficiency. On the other hand, baseline II maximizes the weighted energy efficiency of the system with respect to $W$ and without taking into account the minimum energy harvesting constraint in the optimization problem. The average energy efficiency of the system is computed according to (4) and averaged over 1,000 independent random realizations of multipath fading and path loss attenuation.

Figure 3 shows the average system energy efficiency versus the maximum transmit power allowance for different algorithms. It can be seen that the average system energy efficiency of our proposed algorithms and baseline II is a monotonically non-decreasing function of the maximum transmit power constraint $P$. In particular, the energy efficiency first quickly increases with an increasing $P$ and then saturates when $P>20 \mathrm{dBm}$ due to the fact that a balance between the system energy efficiency and the power consumption of the system is obtained. In the lower transmit power constraint region, such as $5<P<15 \mathrm{dBm}$, all algorithms achieve the same performance in terms of the system energy efficiency criterion. It is also observed that in the low transmit power regime, the system with power splitting receivers achieves a small performance gain compared to the system without energy harvesting receivers since the received power of the desired signal at the receivers may not be sufficiently large for simultaneous information decoding and energy harvesting. However in the high transmit power region, proposed algorithm II outperforms the proposed algorithm I due to the fact that the former is designed based on the optimization of the power splitting factor. Compared to baseline II, about a $6 \%$ gain in terms of energy efficiency can be achieved by algorithm II. In addition, baseline I which aims to maximize the weighted sum rate with respect to the beamformers $W$ achieves a very low energy efficiency due to the fact that the gain of system sum rate cannot compensate the consumption of the transmit power. From the comparison in terms of system capacity as illustrated in Figure 4, some opposite conclusions are obtained.

Figure 5 shows the average system energy efficiency versus the maximum transmit power allowance for proposed algorithm II with different minimum required power transfer. Numerical results reveal that the system energy efficiency does not necessarily increase with an increasing minimum required power transfer. Combining with numerical results observed in Figure 3, it is easy to know that there is an optimal value of minimum required power transfer in the considered systems which can be optimized that would be studied in our future works. It is worth noting that the joint optimization of the power splitting factors and the minimum required power transfer also has 


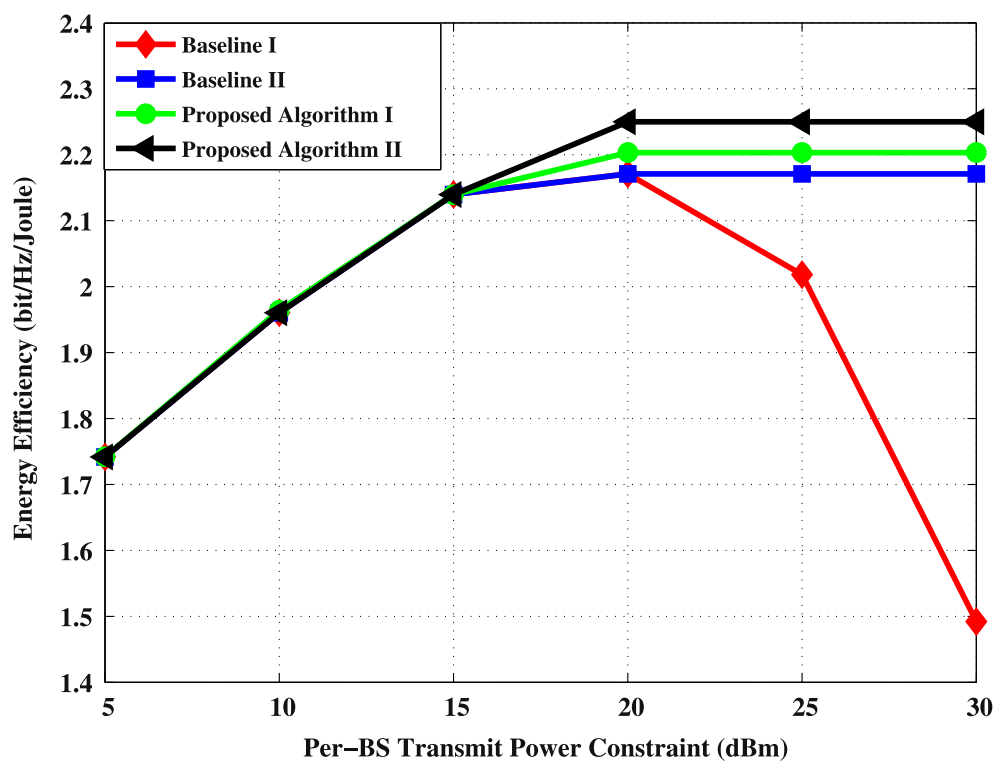

Figure 3 Energy efficiency vs transmit power constraint, $M=4, N=d=I=2, \varrho=0.01 P$.

important affection of the performance of system energy efficiency.

Figure 6 illustrates the average system energy efficiency versus the transmit antenna for proposed algorithm II with a fixed ratio of $M: I$. It can be seen that the system energy efficiency increases with an increasing number of transmit antennas. It also means that the system energy efficiency can be benefited from the user diversity of multiple users. It is interesting to note that these observations are similar to the results obtained in [32], i.e., multiplexing to many users rather than beamforming to a single user and increasing the number of service antennas can simultaneously benefit both the spectral efficiency and the energy efficiency. However, when the number of the transmit antennas is greater than a certain value, i.e., the number of the served users is greater than certain value, the energy efficiency decreases with an increasing number of transmit antennas with a fixed ratio of $M: I$ due to the

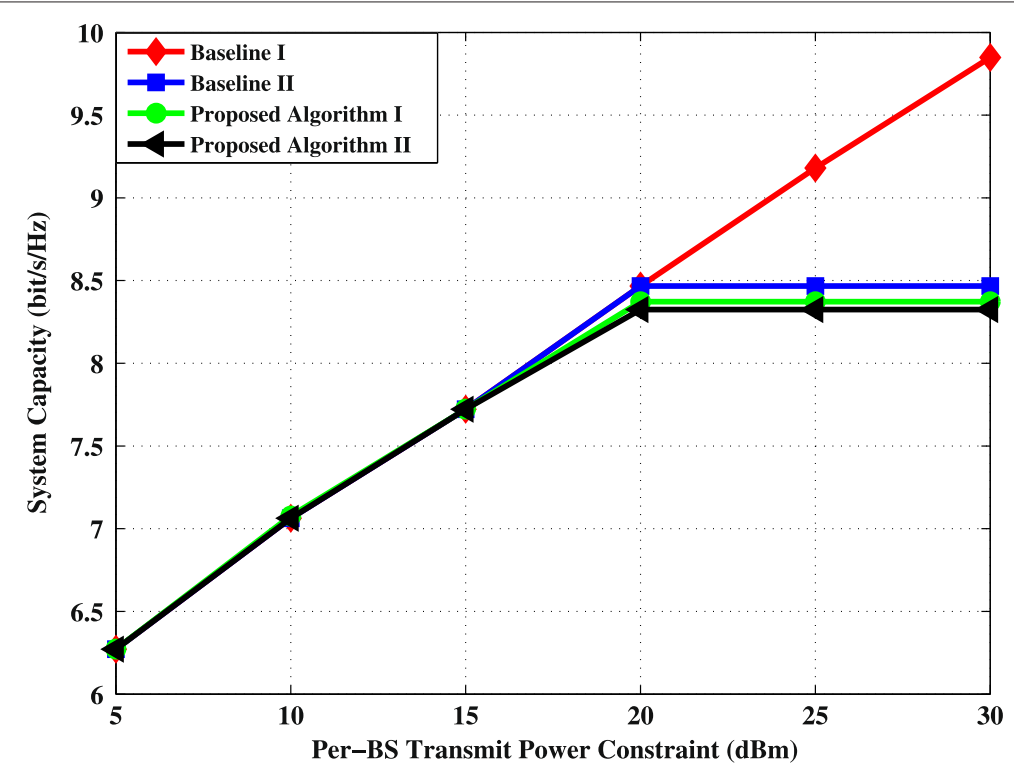

Figure 4 System capacity vs transmit power constraint, $M=4, N=d=I=2, \varrho=0.01 P$. 


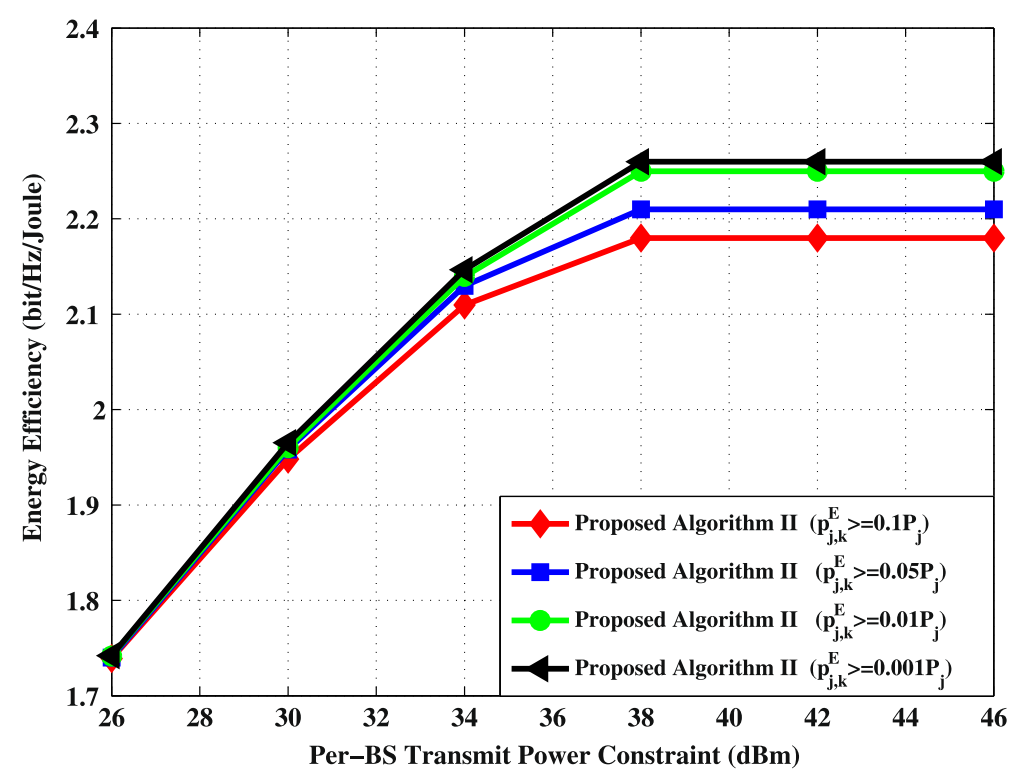

Figure 5 Energy efficiency vs minimum power transfer, $M=4, N=d=I=2$.

fact that the fixed power consumption of the terminal also increases rapidly resulting in a lower energy efficiency. As shown in Figure 7, the energy efficiency of the simulated two algorithms decreases with an increasing fixed receiver power consumption $P_{C_{R}}$.

\section{Conclusions}

In this paper, the transmit beamforming optimization problem was studied for simultaneous wireless infor- mation and power transfer in coordinated multicell multiuser downlink system. Particularly, we focused on the energy efficient precoding design by using the power splitting technique where each receiver divides the received signal into two power streams for concurrent information decoding and energy harvesting. An iterative algorithm to reach its solution was developed by suing the alternating optimization method. Numerical results validate the effectiveness of the proposed algorithm and show that the

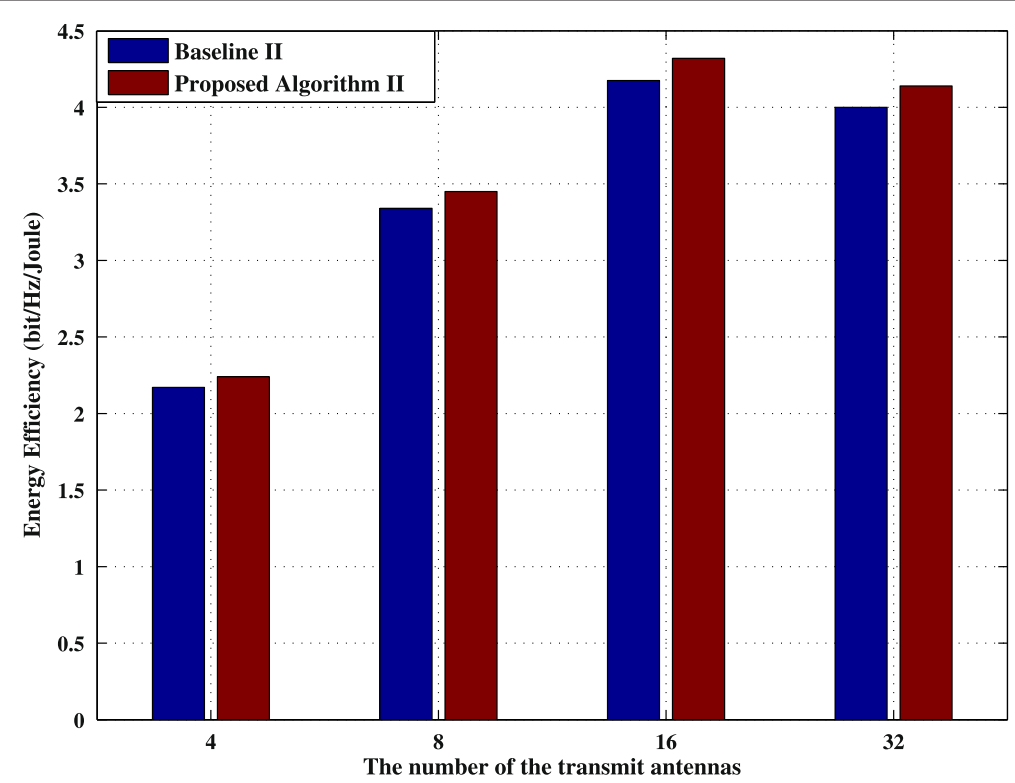

Figure 6 Energy efficiency vs transmit antenna, $M: I=2: 1, N=d=2, P=30 \mathrm{dBm}, \varrho=0.01 P$. 


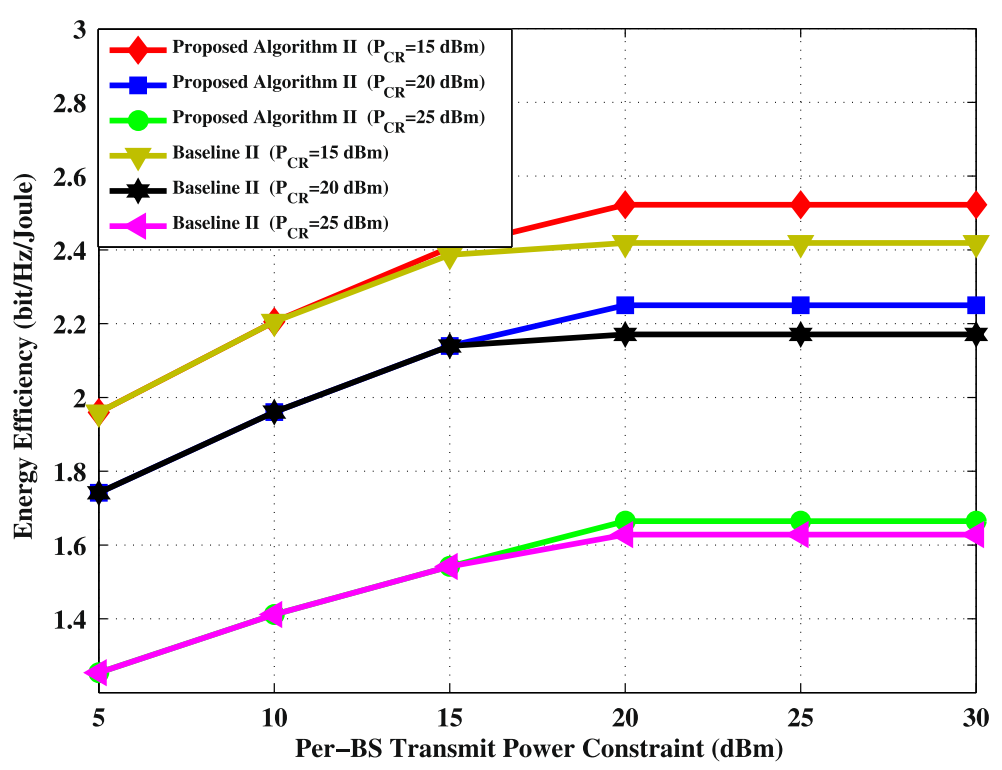

Figure 7 Energy efficiency vs minimum power transfer, $M=4, N=d=I=2, \varrho=0.01 P$.

optimal energy efficiency and spectral efficiency can be achieved simultaneously in the low SNR region; while in the middle-high SNR region, the maximization of spectral efficiency usually cannot bring optimal energy efficiency at the same time and vice versa. Compared to the energy efficiency performance achieved by the traditional energy efficient transmission, the proposed algorithm can achieve better energy efficiency performance.

\section{Endnotes}

a Note that by imposing the power splitting factors $\rho_{j, k}=1, \forall j, k$, then problem (4) reduces to the traditional energy efficient transmission optimization problem. In our paper, the values of $\rho$, where $\rho$ denote the collection of all $\rho_{j, k}$, is first assumed to be fixed in order to obtain an effective solution to the energy efficient precoding problem. We then considered then the power splitting faction optimization for a special case where all users have the same ability to harvest energy. It should be pointed out that the considered problem formulation is also applicable to the case with time-switching receivers (or, under the given time instant, each receiver is either for energy harvesting or information decoding) by adjusting the power splitting factor of each user [11].

${ }^{b}$ We would like to point out that our developed energy efficient transmission algorithm with energy harvesting can also apply to the special case where the value of $\varrho_{j, k}$ is zero, $\forall j, k$.

cNote that for LOS component, we use the far-field uniform linear antenna array model [30].

\section{Competing interests}

The authors declare that they have no competing interests.

\section{Acknowledgements}

This work was supported by National Science and Technology Major Project of China under Grant 2012ZX03004005-003, National Basic Research Program of China under Grant 2013CB329002, National Natural Science Foundation of China under Grants 61471120, 61271018, 61422105, and 61372101, and Research Project of Jiangsu Province under Grants BK20130019 and BK2012021.

Received: 21 October 2014 Accepted: 16 February 2015

Published online: 11 March 2015

\section{References}

1. Z Xu, C Xiong, C Yang, S Zhang, Y Chen, S Xu, Energy efficient wireless communications: tutorial, survey, and open issues. IEEE Wireless Commun. Mag. 18(6), 28-35 (2011)

2. C Han, T Harrold, S Armour, I Krikidis, S Videv, P Grant, H Haas, J Thompson, I Ku, C Wang, T Le, M Nakhai, J Zhang, L Hanzo, Green radio: Radio techniques to enable energy-efficient wireless networks. IEEE Commun. Mag. 49(6), 46-54 (2011)

3. D Feng, C Jiang, G Lim, L Cimini Jr, G Feng, G Li, A survey of energy-efficient wireless communications. IEEE Commun. Surv. Tutorials. 15(1), 167-178 (2013)

4. C Isheden, Z Chong, E Jorswieck, G Fettweis, Framework for link-level energy efficiency optimization with informed transmitter. IEEE Trans. Wireless Commun. 11(8), 2946-2957 (2012)

5. Y Huang, J Xu, L Qiu, Energy efficient coordinated Beamforming for multi-cell MISO systems. IEEE Globecom, 2526-2531 (2013)

6. S He, Y Huang, S Jin, L Yang, Coordinated beamforming for energy efficient transmission in multicell multiuser systems. IEEE Trans. Commun. 61(12), 4961-4971 (2013)

7. S He, Y Huang, L Yang, B Ottersten, Coordinated multicell multiuser precoding for maximizing weighted sum energy efficiency. IEEE Trans. Sig. Proc. 62(3), 741-751 (2014)

8. S He, Y Huang, H Wang, S Jin, L Yang, Leakage-aware energy-efficient beamforming for heterogeneous multicell multiuser systems. EEE J. Sel. Commun. Area. 32(6), 1268-1281 (2014)

9. L Musavian, T Le-Ngoc, Energy-efficient power allocation over nakagami-m fading channels under delay-outage constraints. IEEE J. Sel. Areas Commun. 13(80), 4081-4091 (2014)

10. R Zhang, CK Ho, MIMO broadcasting for simultaneous wireless information and power transfer. IEEE Trans. Wireless Commun. 12(5), 1989-2001 (2013) 
11. J Xu, L Liu, R Zhang, Multiuser MISO beamforming for simultaneous wireless information and power transfer. IEEE Trans. Signal Process. 62(18), 4798-4810 (2014)

12. L Liu, R Zhang, K Chua, Wireless information and power transfer: a dynamic power splitting approach. IEEE Trans. Commun. 61(9), 3990-4001 (2013)

13. STimotheou, I Krikidis, G Zheng, B Ottersten, Beamforming for MISO interference channels with QOS and RF energy transfer. IEEE Trans. Wireless Commun. 13(5), 2646-2658 (2014)

14. Q Shi, WXu L Liu, R Zhang, Joint transmit beamforming and receive power splitting for MISO SWIPT systems. IEEE Trans. Wireless Commun. 13(60), 3269-3280 (2014)

15. X Kang, Y Chia, C Ho, S Sun, Cost minimization for fading channels with energy harvesting and conventional energy. IEEE Trans. Wireless Commun. 13(8), 4586-4598 (2014)

16. X Zhou, R Zhang, CK Ho, Wireless information and power transfer: architecture design and rate-energy tradeoff. IEEE Trans. Commun. 61(11), 4757-4767 (2013)

17. D Ng, E Lo, R Schober, Wireless information and power transfer: Energy efficiency optimization in OFDMA systems. IEEE Trans. Wireless Commun. 12(12), 6352-6370 (2013)

18. R Jagannathan, On some properties of programming problems in parametric form pertaining to fractional programming.Manage. Sci. 12(7), 609-615 (1966)

19. W Dinkelbach, On nonlinear fractional programming. Bull. Aust. Math. Soc. 13(3), 492-498 (1967)

20. J Borwein, A Lewis, Convex analysis and nonlinear optimization: Theory and examples. (Canadian Mathematical Society, Springer Verlag, 2006)

21. S Christensen, R Agarwal, E Carvalho, J Cioffi, Weighted sum rate maximization using weighted MMSE for MIMO-BC beamforming design. IEEE Trans. Wireless Commun. 7(12), 4792-4799 (2008)

22. Q Shi, M Razaviyayn, Z Luo, C He, An iteratively weighted MMSE approach to distributed sum-utility maximization for a MIMO interfering broadcast channel. IEEE Trans. Sig. Proc. 59(9), 4331-4340 (2011)

23. CMeyer, Matrix Analysis and Applied Linear Algebra. (Siam, Society for Industrial and Applied Mathematics Philadelphia, PA, USA, 2000)

24. L Vandenberghe, S Boyd, Semidefinite programming. SIAM Rev 38(1), 49-95 (1996)

25. H Yu, V Lau, Rank-constrained schur-convex optimization with multiple trace/log-det constraints. IEEE Trans. Signal Process. 59(1), 304-314 (2011)

26. M Solodov, On the convergence of constrained parallel variable distribution algorithm. SIAM J. Optim. 8(1), 187-1969 (1998)

27. J Bibby, Axiomatisations of the average and a further generalisation of monotonic sequences. Glasgow Math. J. 15, 63-65 (1974)

28. G Golub, C Van Loan, Matrix Computations, 3rd ed. (The John Hopkins Univ. Press, Baltimore, MD, 1996)

29. X Zhang, J Lee, Low complexity MIMO scheduling with channel decomposition using capacity upperbound. IEEE Trans. Commun. 56(6), 871-876 (2008)

30. E Karipidis, N Sidiropoulos, Z Luo, Far-field multicast beamforming for uniform linear antenna arrays. IEEE Trans. Signal Process. 55(10), 4916-4927 (2007)

31. S Jafar, A Goldsmith, Transmitter optimization and optimality of beamforming for multiple antenna systems. IEEE Trans. Wireless Commun. 3(4), 1165-1175 (2004)

32. H Ngo, E Larsson, T Marzetta, Energy and spectral efficiency of very large multiuser MIMO systems. IEEE Trans. Commun. 61(4), 1436-1449 (2013)

\section{Submit your manuscript to a SpringerOpen ${ }^{\mathcal{O}}$ journal and benefit from:}

- Convenient online submission

- Rigorous peer review

- Immediate publication on acceptance

- Open access: articles freely available online

- High visibility within the field

- Retaining the copyright to your article

Submit your next manuscript at $\boldsymbol{\wedge}$ springeropen.com 